\title{
Orientable and nonorientable minimal surfaces
}

\author{
Jürgen Jost*
}

Abstract. We describe a theory of Morse-Conley type for orientable and nonorientable minimal surfaces of varying topological type solving Plateau problems in $\mathbf{R}^{3}$.

1991 Mathematics Subject Classification 53A10, 49F10, 58E12, 32G15

After J. Douglas had solved Plateau's problem (this was also independently achieved by T. Radó), he considered the problem of finding minimal surfaces of higher genus and/or connectivity bounded by a given configuration of Jordan curves in 3 -space ([D2]). In fierce competition, this problem was also studied by R. Courant and his student Shiffman ([C], [Sh1]). The result stated in these papers can be expressed as follows.

Theorem 1. Let $\Gamma:=\left(\Gamma_{1}, \ldots, \Gamma_{k}\right)$ be a collection of disjoint oriented Jordan curves in $\mathbf{R}^{3}$. Let

$a(\Gamma, g):=\inf$ Area $(\Sigma): \Sigma$ is an oriented surface of genus $g$ in $\mathbf{R}^{3}$ with $\partial \Sigma=\Gamma\}$

$a^{*}(\Gamma, g):=\inf$ Area $\left(\Sigma^{\prime}\right): \Sigma^{\prime}$ is either an oriented surface of genus $<g$ or a union of at least two surfaces with sum of genera $\leq g$ in $\mathbf{R}^{3}$ and $\left.\partial \Sigma=\Gamma\right\}$. If $a(\Gamma, g)<a^{*}(\Gamma, g)$, then $\Gamma$ bounds an oriented minimal surface of genus $g$.

The condition $a(\Gamma, g)<a^{*}(\Gamma, g)$ is refered to as Douglas condition. An important insight of Douglas was that instead of minimizing area it is much more convenient to minimize Dirichlet's integral

$$
D(h, \Sigma):=\frac{1}{2} \int_{\Sigma}\left(h_{u}^{2}+h_{v}^{2}\right) d u d v
$$

for maps $h: \Sigma \rightarrow \mathbf{R}^{3}$ from a Riemann surface $\Sigma$ mapping $\partial \Sigma$ monotonically and with preserved orientation onto $\Gamma$. Here, $w=u+i v$ is a local conformal

The author gratefully acknowledges the support of the German Science Foundation (DFG) during the period that this research was carried out. 
parameter on $\Sigma$. Actually, since for any $\eta \in H^{\frac{1}{2}}(\partial \Sigma, \Gamma)$, the harmonic extension $h=h_{\eta}:=\Sigma \rightarrow \mathbf{R}^{3}$ is unique and minimizes $D$ with respect to its own boundary values $\eta=h_{\mid \partial \Sigma}$, one then studies

$$
E(\eta, \Sigma):=\frac{1}{2} \int_{\Sigma}\left(h_{u}^{2}+h_{v}^{2}\right) d u d v
$$

with $h=h_{\eta}$.

One then has to vary $E$ over all boundary maps $\eta$ and over all conformal structures of surfaces of genus $g$. A key step in the proof then consists in showing that if one has a minimizing sequence with the property that the sequence of underlying conformal structures does not contain a convergent subsequence, then the Douglas condition is violated.

Since at the time of these works, the geometry of the moduli space $\mathcal{M}_{g}$ of Riemann surfaces of genus $g$ was not understood and since in particular the MumfordDeligne compactification $\overline{\mathcal{M}}_{g}$ was not available, at this point the arguments were not completely satisfactory, at least at the conceptual level. Tromba [T1] pointed this out, revived interest in the theory of minimal surfaces of higher genus, and in collaboration with A. Fischer reworked Teichmüller theory to make it more suitable for applications in minimal surface theory (see [T2]). A modern proof of Thm. 1 was first achieved in [J1] (see also [TT] for a similar argument in a different conceptual setting).

One may also ask about nonorientable minimal surfaces bounded by suitable configurations of disjoint, smooth Jordan curves, now without orientation. This case was also treated inDouglas' classical paper [D2], with an earlier treatment of the Möbius strip in [D1]. Recently, a treatment along the lines of [J1] was given by $\mathrm{F}$. Bernatzki $[\mathrm{Be}]$. In order to formulate the result, we define the genus of a nonorientable surface to be $g$ if it has a two sheeted orientable covering of genus $2 g-1$. This is different from the traditional definition of genus in the nonorientable case and in particular allows the genus to take half integer values, but it will facilitate the transition between surfaces of different topological structure below. The result then becomes

Theorem 2. Let $\Gamma=\left(\Gamma_{1}, \ldots, \Gamma_{k}\right)$ be a collection of disjoint Jordan curves in $\mathbf{R}^{3}$. Let

$a(\Gamma, g, n):=\inf \{$ Area $(\Sigma): \Sigma$ is a nonorientable surface of genus $<g$ in $\mathbf{R}^{3}$ with $\left.\partial \Sigma=\Gamma\right\}$

$a^{*}(\Gamma, g, n):=\inf \left\{\right.$ Area $\left(\Sigma^{\prime}\right): \Sigma^{\prime}$ is either a surface - orientable or nonorientable - of genus $g$ or a union of at least two surfaces - orientable or nonorientable - with sum of genera $\leq g$ in $\mathbf{R}^{3}$ with $\left.\partial \Sigma=\Gamma\right\}$.

If $a(\Gamma, g, n)<a^{*}(\Gamma, g, n)$, then $\Gamma$ bounds a nonorientable minimal surfaces of genus $g$.

Corollary 1. Suppose $a^{*}(\Gamma, g)$ is realized by a connected nonembedded minimal surface. Then $\Gamma$ bounds an oriented minimal surface of genus $g$. 
Proof. By regularity results for minimal surfaces, a nonembedded minimal surface $\Sigma^{\prime}$ contains an open Jordan arc $\gamma$ along which locally two (or more) embedded sheets $S_{1}, S_{2}$ of the surface meet transversally. We take (sufficiently small) closed Jordan curves $c_{1}, c_{2}$ on $S_{1}$ and $S_{2}$, resp., with $c_{1} \cap \gamma=c_{2} \cap \gamma=c_{1} \cap c_{2}=\left\{z_{1}, z_{2}\right\}$. $z_{1}$ and $z_{2}$ divide $c_{i}$ into two subarcs $c_{i}^{1}, c_{i}^{2}, i=1,2$, and $\gamma$ and $c_{i}^{j}$ bound a region $S_{i}^{j}$ on $S_{i}(i, j=1,2)$. Then $c_{1}^{1} \cup c_{2}^{1}$, e.g., is a closed Jordan curve and bounds an area minimizing disk $S_{12}^{11}$ by the solution of Plateaus's problem. Since $S_{1}^{1} \cup S_{2}^{1}$ is not smooth along $\gamma$, it cannot be area minimizing by the regularity of area minimizing disks. Hence

$$
\operatorname{Area}\left(S_{12}^{11}\right)<\operatorname{Area}\left(S_{1}^{1} \cup S_{2}^{1}\right),
$$

and similarly for $c_{1}^{1} \cup c_{2}^{2}, c_{1}^{2} \cup c_{2}^{1}, c_{1}^{2} \cup c_{2}^{2}$. We thus can decrease the area of $\Sigma^{\prime}$ by replacing $S_{1}^{1} \cup S_{1}^{2} \cup S_{2}^{1} \cup S_{2}^{2}$ by either $S_{12}^{11} \cup S_{12}^{22}$ or by $S_{12}^{12} \cup S_{12}^{21}$. We thus have replaced the union of two disks by a handle. Consequently, the genus is increased by 1 . One of the two new surfaces is orientable, the other one nonorientable. Taking the orientable one, we conclude that its genus cannot be smaller than $g$ because $\Sigma^{\prime}$ realizes $a^{*}(\Gamma, g)$, and we thus verify the Douglas condition

$$
a(\Gamma, g)<a^{*}(\Gamma, g)
$$

and apply Theorem 1 .

The preceding argument shows that one can also decrease the area of a nonembedded minimal surface by adding a nonorientable handle. A modification of the argument, however, shows that one may in this case already decrease area by inserting a Möbius strip. We therefore also obtain

Corollary 2. Suppose $a^{*}(\Gamma, g, n)$ is realized by a nonembedded connected minimal surface. Then $\Gamma$ bounds a nonorientable minimal surface of genus $g$.

It is an interesting question which configurations of Jordan curves can bound infinitely many minimal surfaces. Courant and P. Levy first suggested an example of a rectifiable Jordan curve which is smooth except at one point and which bounds infinitely many minimal surfaces. To make this example regorous, one needs the bridge theorem which was only proved much later by Meeks-Yau [MY]. Here we observe an example which is similar in spirit but does not need the bridge theorem. We define the genus of a Jordan curve $\Gamma$ in $\mathbf{R}^{3}$ as the smallest $g$ for which $\Gamma$ bounds an embedded surface of genus $g$. (One may then specify in addition whether the surface is orientable or not.) If there is no such finite $g$, we define the genus to be $\infty$. It is easy to construct rectifiable Jordan curves which are smooth except at one point and of genus $\infty$. Namely one starts with any Jordan curve of positive genus and adds infinitely many copies of this curve (in the sense of addition of knots), but scales them down successively in length so that the final curve has finite length.

Corollary 3. Any rectifiable Jordan curve of infinite genus bounds infinitely many (orientable and nonorientable) minimal surfaces. 
Proof. By the arguments for the proofs of Cor. 1,2, the Douglas conditions are satisfied for any $g$.

It is an open question whether there exists a single smooth Jordan curve in $\mathbf{R}^{3}$ that is capable of bounding infinitely many minimal surfaces. Morgan [Mo] constructed a configuration of 4 disjoint smooth Jordan curves bounding infinitely many minimal surfaces, and later Gulliver-Hildebrandt [GH] and Pitts [P] gave an example with three such curves. (The configuration of $[\mathrm{GH}]$ and $[\mathrm{P}]$ is the same but the constructed minimal surfaces are different.) In both cases, all curves are circles in parallel planes, and there have to be infinitely many, because any irreducible embedded minimal surfaces with this boundary cannot respect all the rotational symmetries of the boundary configuration. We here want to present a construction with only two curves which at the same time is more general than the preceding ones.

Theorem 3. Let $\Gamma_{1}$ and $\Gamma_{2}$ be two smooth, oriented, disjoint Jordan curves in $\mathbf{R}^{3}$ which are linked. Assume that there exists an involution $i$ (i.e. an isometry of order 2) of $\mathbf{R}^{3}$ leaving $\Gamma_{1}$ pointwise fixed and mapping $\Gamma_{2}$ onto itself but reversing its orientation. Then the configuration $\Gamma=\left(\Gamma_{1}, \Gamma_{2}\right)$ bounds infinitely many nonorientable minimal surfaces.

Examples of curves satisfying the assumptions are given by suitable linked circles in orthogonal planes.

Theorem 3 may be deduced from the general result of Thm. 4 below. Let us describe some of the general constructions needed. We first need to display the geometry of the moduli and Teichmüller spaces for oriented and nonorientable surfaces. (A reference for the oriented case is [A].) For simplicity, we shall describe the relevant constructions only for closed surfaces although for Plateau's problem we need Riemann surfaces with boundary. Taking Schottky doubles of those i.e. reflecting them across their boundary, reduces their treatment to the case of closed ones.

We only treat the case of genus $g>1$ since the other cases are simpler. Thus, all surfaces are covered by the upper half plane and carry hyperbolic metrics. A nonorientable surface can also carry a conformal structure in the sense that it has an atlas of coordinate charts with holomorphic or antiholomorphic transition functions. All concepts from complex analysis can either be expressed w.r.t. this structure or else on the two-sheeted orientable cover of genus $2 g-1$.

Fenchel-Nielsen coordinates on Teichmüller space $T_{g}$ for oriented surfaces of genus $g$ are defined by cutting such a surface into three-circle-domains, also called pairs of pants, with geodesic boundary curves. The lengths of these $3 g-3$ curves give positive parameters $\left(\ell_{1}, \ldots, \ell_{3 g-3}\right)$. Twist parameters obtained by identifying pairs of boundary curves of such three-circle-domains in order to reglue the surface yield the remaining coordinates $\left(\theta_{1}, \ldots, \theta_{3 g-3}\right)$ with values in $\mathbf{R}$. Geometric degeneration of elements in $T_{g}$ is characterized by simple closed geodesics shrinking to zero in length. In suitable Fenchel-Nielsen coordinates, in this case one or several of the $\ell_{i}$ tend to zero - for simplicity of exposition, we assume that only one $\ell_{i}$ does. In the limit, one obtains a surface of genus $g-1$ with two nodes 
corresponding to the pinched geodesic. Also, the corresponding twist parameter $\theta_{i}$ becomes undetermined if $\ell_{i}=0$. It is now natural to also allow $\ell_{i}$ to become negative and letting this correspond to glueing the two geodesic boundary curves of length $\left|\ell_{i}\right|$ of our three-circle-domains with opposite orientation as before, thus creating a nonorientable handle instead of an orientable one. (We note, however, that the assignement of the twist parameter $\theta_{i}$ across $\ell_{i}=0$ is only determined up to the choice of a shift $\tau \in \mathbf{R}$.) Vice versa, it is also a possible type of geometric degeneration of a nonorientable surface to pinch an orientable or nonorientable handle by letting its core geodesic shrink to zero. Here, however, also another type of degenration occurs, namely pinching the core geodesic of a Möbius strip. The length of such a geodesic also gives a length parameter on the corresponding Teichmüller space, but in this case we do not have an associated twist parameter.

We can also pass to corresponding moduli spaces by identifying conformally equivalent surfaces. In order to have a consistent construction, in the oriented case we also have to identify surfaces if there exists an antiholomorphic bijection between them. Thus, the moduli space occuring here is obtained from the standard one by dividing out an involution. On the moduli space level, one reduces the indeterminacy of the twist parameters at the boundary so that here the boundary has complex codimension 1 instead of real codimension 1. Again, also in this level one can go across the boundary from orientable to nonorientable surfaces. The proper enlarged moduli space $\overline{\overline{\mathcal{M}}}_{g}$ then includes all orientable and nonorientable surfaces of genus $\leq g$, those of genus $<g$ occuring with additional cusps or punctures or nodes. For surfaces with $k$ boundary curves, the corresponding enlarged moduli space is denoted by $\overline{\overline{\mathcal{M}}}_{g, k}$.

With this framework we can also describe a direct proof of Thm. 3. It is based on a symmetry argument. (Such a symmetry argument has been used by J. Pitts $[\mathrm{P}]$ to show the existence of infinitely many oriented minimal surfaces of increasing topological type bounded by three parallel circles.) $\Gamma_{1}$ and $\Gamma_{2}$ each bound a minimal disk. For simplicity, we assume that they are unique. Therefore, they are invariant under the involution $i$. These two disks together then form a minimal surface $\Sigma_{0}$ bounded by $\Gamma$. Since $\Gamma_{1}$ and $\Gamma_{2}$ are linked, the minimal disks have to intersect and so $\Sigma_{0}$ is not embedded. By Cor. $1, \Gamma$ therefore also bounds a minimal annulus $\Sigma_{1}$. Since $\Sigma_{1}$ has only one component, it cannot be invariant under $i$, and $i\left(\Sigma_{1}\right)$ then is another minimal surface bounded by $\Gamma$. The conformal structures of $\Sigma_{1}$ and $i\left(\Sigma_{1}\right)$ can be connected in the class of nonorientable surfaces of genus 1 (Klein bottles with two holes), i.e. in $\overline{\bar{M}}_{1,2}$. We distinguish two cases:

1) $\Sigma_{1}$ and $i\left(\Sigma_{1}\right)$ are area minimizing among surfaces with conformal structures in $\overline{\overline{\mathcal{M}}}_{1,2}$. We minimax, i.e. consider

$$
\begin{aligned}
\kappa_{1}:=\inf _{\gamma} \sup _{t \in I}\{ & E(\eta(t), S(t)): \gamma(t)=(\eta(t), S(t)), t \in[0,1], \\
& \text { is a continuous path in (boundary maps) } x \overline{\bar{M}}_{1,2} \\
& \text { with } \left.\gamma(0)=\Sigma_{1}, \gamma(1)=i\left(\Sigma_{1}\right)\right\} .
\end{aligned}
$$


One shows that $\kappa_{1}$ is realized by a minimal surface $\Sigma_{2}$ different from $\Sigma_{1}$ and $i\left(\Sigma_{1}\right) . \Sigma_{2}$ is also different from $\Sigma_{0}$. Namely, $\Sigma_{2}$ should be of critical index at most 1 , whereas one can insert arbitrarily many disjoint handles along the line of self intersection of $\Sigma_{0}$ as in the proof of Cor. 1 and therefore decrease its area in infinitely many independent ways so that $\Sigma_{0}$ should have infinite index if one allows arbitrary genus and at least index 2 if one allows genus 2. In particular, no critical path $\gamma$ can pass through $\Sigma_{0}$. Therefore, $\Sigma_{2}$ is not invariant under $i$, and $i\left(\Sigma_{2}\right)$ is another minimal surface bounded by $\Gamma$ and different from all the preceding ones.

2) If $\Sigma_{1}$ and $i\left(\Sigma_{1}\right)$ are not area minimizing among surfaces of class $\overline{\overline{\mathcal{M}}}_{1,2}$, we let $\Sigma_{2}$ be the minimizing surfaces in this class. Again, it is different from $\Sigma_{0}$, and so $i\left(\Sigma_{2}\right)$ is another minimal surface bounded by $\Gamma$.

The process can be iterated with additional parameters. Since except for $\Sigma_{0}$, all occuring minimal surfaces are not invariant under $i$, they always occur in pairs, and this then always allows higher order saddle point constructions. The crucial point is that $\Sigma_{0}$ is of infinite index and can therefore never occur as a solution of a finite order saddle point construction.

So much for a sketch of the proof of Thm. 3. It points towards a general ciritcal point theory for minimal surfaces of varying topological type and orientability character, and we now want to briefly describe that theory.

One wants to use $E$ as a Morse function. The main achievement of [J1] was a construction showing the continuity of $E$ under variation of topological type (going from the interior of moduli space to its boundary). This was then refined in [JS] to show even smoothness of $E$ under variation of topological type (see [S3] for a technical correction), and when combined with Morse theoretic constructions for minimal surfaces of disk or annulus type from [S1], [S2] (earlier Morse theoretic results for minimal surfaces are in [MT1], [MT2], [Sh2],[Sh3], and the revivement of the subject is again due to Tromba) and recent extensions of Conley's theory, it yielded a general Morse-Conley theory for oriented minimal surfaces.

In [JS], $E$ and its derivatives were computed in Fenchel-Nielsen coordinates. It is possible, however, to carry out the computations also in complex coordinates on the moduli spaces, and the resulting formulae fit nicely together with the WeilPetersson geometry of $\overline{\overline{\mathcal{M}}}_{g, k}$.

For an embedded minimal surface of genus $g$, as in [JS], its critical index when computed in $\overline{\overline{\mathcal{M}}}_{g^{\prime}, k}$, is independent of $g^{\prime} \geq g$ (except for the degeneracy introduced by the node parameters of the boundary strata, an issue addressed below). If the minimal surface is nonembedded, its index becomes infinite if we allow surfaces of arbitrary genus, and so it does not appear in the final Morse-Conley relations. This issue has not been treated entirely correctly in [JS]. Also, for technical reasons, the computations are carried out on the Teichmüller space level in order to avoid singularities. This introduces certain redundancies, however. First, one has invariance under the discrete action of the modular group. Second, at the boundary, one has invariance under the twist parameters as described above. Third, even on the moduli space level, one has the redundancy that boundary elements represent 
surfaces with nodes and thus carry additional parameters for the position of the nodes. A bounded harmonic function on a Riemann surface, however, smoothly extends through an isolated singularity, and therefore, the position of the nodes is irrelevant for the resulting minimal surface. Therefore, the correct space on which to compute Conley indices for minimal surfaces is obtained form $\overline{\overline{\mathcal{M}}}_{g, k}$ by collapsing the directions corresponding to the node parameters in the boundary components. The resulting space is highly singular (it is a generalization of Baily's compactification of $\mathcal{M}_{g}$ [Ba]). There is, however, a general strategy to carry out Conley theory on stratified singular spaces, assuming that the indices of critical points do not change under restriction to lower dimensional strata. Let $X$ be a stratified singular space, and let $\pi: \hat{X} \rightarrow X$ be a resolution of singularities. A functional $F: X \rightarrow \mathbf{R}$ lifts to $\hat{F}=F \circ \pi: \hat{X} \rightarrow \mathbf{R}$, and one then needs to construct a $\pi$-invariant (pseudo)gradient flow $\Phi: \hat{X} \times \mathbf{R}_{+} \rightarrow \hat{X}$. Thus, if $\pi(x)=\pi(y)$, one requires that also $\pi(\Phi(x))=\pi(\Phi(y))$. If certain general assumptions of Conley theory are met, one finds index pairs $(\mu, \nu)$ on $X$ (meaning that $\pi^{-1}(\mu, \nu)$ is an index pair on $\hat{X}$ ), and the Conley index of a critical point $x_{0}$ of $F$ is the power series

$$
i_{t}\left(x_{0}\right)=\Sigma_{p=0}^{\infty} \operatorname{dim} H^{q}(\mu, \nu) t^{q}
$$

where $(\mu, \nu)$ is an index pair for $x_{0}$, and we take $\mathbf{Z}_{2}$-cohomology. If $x_{0}$ is a nondegenerate critical point in the sense of Morse theory of Morse index $m_{0}$, then

$$
i_{t}\left(x_{0}\right)=t^{m_{0}} \text {. }
$$

In conclusion, one obtains for example similarly as in [JS]

Theorem 4. Suppose $\Gamma$ bounds only finitely many orientable and nonorientable minimal surfaces $\Sigma_{j}$ of finite genus. Then

$$
\sum_{j=1} i_{t}\left(\Sigma_{j}\right)=1+(1+t) Q(t),
$$

where $Q(t)$ has nonnegative integer coefficients, and the sum is only over the embedded ones among the $\Sigma_{j}$.

Morse theoretic conclusions follow in an obvious manner.

Details will be presented in a different publication. For the definition of certain technical terms (whose intuitive meaning should be clear, however), we refer to [J2] or [JS].

The author thanks M. Struwe for his comments.

\section{References}

[A] Abikoff, W., The real analytic theory of Teichmüller space. Springer Lect. Notes Math. 820 (1980)

[Ba] Baily, W.L., Jr., On the moduli of Jacobian varieties. Ann. Math. 71 (1960), 303-314

[Be] Bernatzki, F., Nichtorientierbare Minimalflächen. Diplomarbeit, Bochum, 1991 
[C] Courant, R., The existence of minimal surfaces of given topological structure under prescribed boundary conditions. Acta Math. 72 (1940), 51-98

[D1] Douglas, J., One sided minimal surfaces with a given boundary. Trans. AMS 34 (1932), 731-756

[D2] Douglas, J., Minimal surfaces of higher topological structure. Ann. Math. 40 (1939), 205-298

[GH] Gulliver, R., and S. Hildebrandt, Boundary configurations spanning continua of minimal surfaces. Manuscr. Math. 54 (1986), 323-347

[J1] Jost, J., Conformal mappings and the Plateau-Douglas problem in Riemannian manifolds. J. Reine \& Angew. Math. 359 (1985), 37-54

[J2] Jost, J., Two Dimensional Geometric Variational Problems. John Wiley - Interscience, Chichester, 1991

[JS] Jost, J., Struwe, M., Morse-Conley theory for minimal surfaces of varying topological type. Inv. math. 102 (1990), 465-499

[MY] Meeks, W., Yau, S.T., The existence of embedded minimal surfaces and the problem of uniqueness. Math. Z. 179 (1982), 151-168

[Mo] Morgan, F., A smooth curve in $\mathbf{R}^{3}$ bounding a continuum of minimal surfaces. Arch. Rat. Mech. Anal. 75 (1980), 193-197

[MT1] Morse, M., Tompkins, C., The existence of minimal surfaces of general critical types. Ann. Math. 40 91939), 443-472 and Ann. Math. 41 (1941), 331

[MT2] Morse, M., Tompkins, C., Unstable minimal surfaces of higher topological structure. Duke Math. J. 8 (1941), 350-375

[P] Pitts, J., private communication

[Sh1] Shiffman, M., The Plateau problem for surfaces of arbitrary topological structure. Amer. J. Math. 61 (1939), 853-882

[Sh2] Shiffman, M., The Plateau problem for nonrelative minima. Ann. Math. 40 (1939), 834-854

[Sh3] Shiffman, M., Unstable minimal surfaćes with several boundaries. Ann. Math. 43 (1942), 197-222

[S1] Struwe, M., On a critical point theory for minimal surfaces spanning a wire in $\mathbf{R}^{n}$. J. Reine \& Angew. Math. 349 (1984), 1-23

[S2] Struwe, M., A Morse theory for annulus type of minimal surfaces. J. Reine \& Angew. Math. 368 (1986), 1-27

[S3] Struwe, M., Minimal surfaces of higher genus and general critical type. to appear in Proc Nankai Inst.

[TT] Tomi, F., Tromba, A., Existence theorems for minimal surfaces of non-zero genus spanning a contour. Memoirs AMS 71, no. 382 (1988)

[T1] Tromba, A., On Plateau's problem for minimal surfaces of higher genus in $\mathbf{R}^{n}$.

[T2] Tromba, A., Global analysis and Teichmüller theory. In: Seminar on new results in nonlinear PDE, (ed by Tromba, A.), Vieweg, Braunschweig, Wiesbaden, 1987 